\title{
Volatile Removal Assembly Flight Experiment and KC-135 Packed Bed Experiment: Results and Lessons Learned
}

Donald W. Holder
NASA, Marshall Space Flight Center

David S. Parker Hamilton Sundstrand on Environmental Systems Toulouse, France July $10-13,2000$ 
SAE routinely stocks printed papers for a period of three years following date of publication. Direct your orders to SAE Customer Sales and Satisfaction Department.

Quantity reprint rates can be obtained from the Customer Sales and Satisfaction Department.

To request permission to reprint a technical paper or permission to use copyrighted SAE publications in other works, contact the SAE Publications Group.

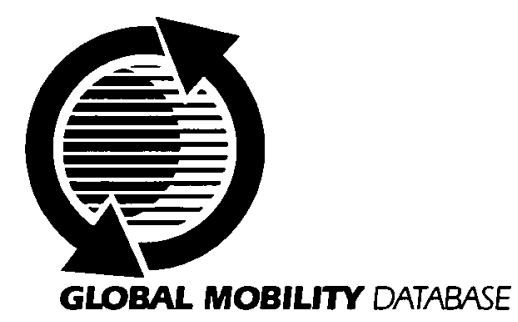

All SAE papers, standards, and selected books are abstracted and indexed in the Global Mobility Database

\section{ISSN 0148-7191}

Positions and opinions advanced in this paper are those of the author(s) and not necessarily those of SAE. The author is solely responsible for the content of the paper. A process is available by which discussions will be printed with the paper if it is published in SAE Transactions. For permission to publish this paper in full or in part, contact the SAE Publications Group.

Persons wishing to submit papers to be considered for presentation or publication through SAE should send the manuscript or a 300 word abstract of a proposed manuscript to: Secretary, Engineering Meetings Board, SAE.

\section{Printed in USA}




\title{
Volatile Removal Assembly Flight Experiment and KC-135 Packed Bed Experiment: Results and Lessons Learned
}

\author{
Donald W. Holder \\ NASA, Marshall Space Flight Center
}

David S. Parker

Hamilton Sundstrand

\begin{abstract}
The Volatile Removal Assembly (VRA) is a high temperature catalytic oxidation process that will be used as the final treatment for recycled water aboard the International Space Station (ISS). The multiphase nature of the process had raised concerns as to the performance of the VRA in a micro gravity environment. To address these concerns, two experiments were designed. The VRA Flight Experiment (VRAFE) was designed to test a full size VRA under controlled conditions in micro gravity aboard the SPACEHAB module and in a $1-\mathrm{g}$ environment and compare the performance results. The second experiment relied on visualization of two-phase flow through small column packed beds and was designed to fly aboard NASA's micro gravity test bed plane (KC-135). The objective of the KC-135 experiment was to understand the twophase fluid flow distribution in a packed bed in micro gravity. On Space Transportation System (STS) flight 96 (May 1999), the VRA FE was successfully operated and in June 1999 the KC-135 packed bed testing was completed. This paper provides an overview of the experiments and a summary of the results and findings.
\end{abstract}

\section{BACKGROUND}

During the early 1990s the Water Processor Assembly (WPA) was being developed by Hamilton Sundstrand under a contract from Boeing Aerospace for eventual installation into the ISS. During the WPA's development the VRA was identified as having possible sensitivity to micro gravity operation. This subassembly was identified as a result of requiring operation in a two-phase, gas/iquid, micro gravity environment. Since the effects of two-phase flow in a packed bed operated in micro gravity are not well understood a risk mitigation plan was developed which included the development of a flight experiment.
A simplified schematic of the VRA is shown in Figure 1. The VRA subassembly performs the final unit operation for polishing the water to potable quality by removing residual organics and microorganisms. Within the VRA, residual organics and microorganisms are oxidized by gaseous oxygen in the presences of a solid phase catalyst and at a temperature of 250-280 F (catalytic oxidation process). Excess oxygen and oxidation byproducts are subsequently removed from the water by phase separation and ion exchange. Regenerative heat exchange is utilized to enhance the energy efficiency of the high temperature process.

Figure 1. VRA Functional Schematic

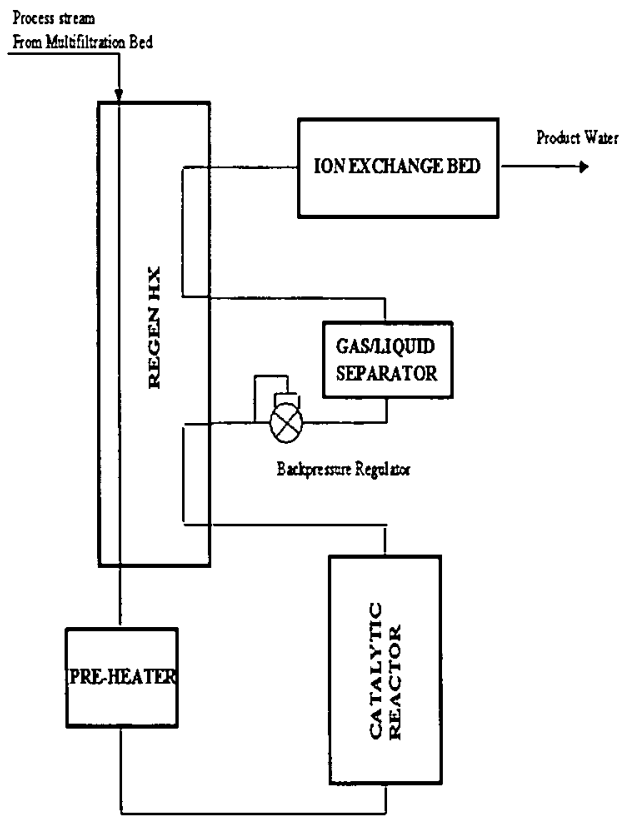

A development contract was issued to Hamilton Sundstrand by NASA Marshall Space Flight Center (MSFC) in July of 1995 to develop a Volatile Removal 
Assembly Flight Experiment (VRAFE). This experiment would be designed to be a closed loop selfcontained/controlled assembly that would be installed on board the SPACEHAB module for micro gravity performance evaluation.

The VRAFE was delivered in June of 1997 to NASA MSFC. NASA MSFC then completed the ground evaluation and baseline performance testing of the experiment prior to the installation in the SPACEHAB.

The first flight of the VRAFE was in January of 1998, on board STS-89. The experiment had been designed to operate continuously for four days. During which time the VRAFE would be monitored on the ground via downlink data while the crew would be taking a series of water sample for later ground analysis. The combination of the on-orbit ground linked data and water sample analysis would then be compared to the previous ground baseline performance results to determine any micro gravity effects.

During the STS-89, an anomaly occurred in the VRAFE after operating for only 102 minutes. The anomaly, which caused an automatic shutdown of the experiment, was the result of a high-pressure safety limit being exceeded. A second attempt at restarting the experiment resulted in the similar shutdown. No further restarts were attempted on the mission. Investigation of the experiment indicated that the high-pressure anomaly occurred as a result of large catalyst particles, coming from the VRA, interfered with the operation of the backpressure regulator. In addition, the Gas Liquid Separator (GLS) was found to contain catalyst particles.

Following the investigation a new risk mitigation plan was developed. Since it was not known at the time the plan was developed if a second flight experiment would occur additional methods were developed for evaluation of the VRA. These methods included a two phased flow analysis within the VRA catalyst bed, flow visualization tests of the catalyst bed, operation of a catalyst bed flow test on board NASA's micro gravity test bed (commonly referred to as the KC-135), and if available a second micro gravity flight. In addition, methods for control of particle generation were developed.

Eventually a second micro gravity flight was scheduled and the experiment was upgraded. This included a new GLS and filters, which would minimize catalyst particles. Two types of filters were used to eliminate the VRA particles, a temporary start up and a permanent in line. The start-up filter was a 0.5 micron filter, which was used to eliminate any particles generated in the VRA during launch. This filter was removed after approximately one hour of system operation, which allowed the particles to be flushed from the reactor. The second filter was installed permanently in the experiment to provide continuous 70 micron filtration down stream of the VRA. Once the upgrades were completed, a series of new ground baseline tests were conducted by MSFC for eventual comparison to the flight results.
The second flight of the VRAFE was in May of 1999, on board STS-96 SPACEHAB Mission. Due to the limited availability of crew time, the experiment was only operated for 32 hours with a total of six water samples taken.

Following the initial flight of the VRAFE, additional activities were initiated to develop a further understanding into the VRA reactor micro gravity operation. These efforts included calculations on gas flow within a packed bed and flow visualization testing. The initial calculations indicated significant gas inclusion could occur which would affect the VRA performance. In order to confirm or disprove the prediction a series of flow visualization tests were conducted. These included tests on the ground and on board the NASA KC135 aircraft with various packed bed VRA reactors.

Detailed descriptions of the VRAFE and KC-135 tests and their results are given in the following sections.

\section{TEST OBJECTIVES AND DESCRIPTIONS}

Within the VRA, multiphase conditions exist throughout much of the assembly. These conditions create the potential for micro gravity to influence the performance of the VRA. Specifically, micro gravity could have a negative affect on the VRA packed-bed, catalytic reactor and REGEN HX.

The most critical component of the VRA, and perhaps the most sensitive to gravity influences, is the packedbed catalytic reactor. Ground tests of VRAs operated in various orientations have shown that overall reactor performance can be influenced by gravity forces. Proper oxidation of trace organics within the reactor requires the intimate mixing and uniform distribution of gaseous oxygen and liquid water throughout a bed of solid catalyst material. Uneven distribution of the gas and liquid phases of the reactor can reduce VRA performance in several ways. First, if large pockets of gas accumulate within the reactor bed, occluding the majority of the reactor with gas, liquid channeling could occur, and inadequate residence time would be achieved within the reactor. Poor distribution of the gas can also cause oxygen-deficiency in areas of the reactor, resulting in incomplete oxidation of contaminants. Through $1 \mathrm{gg}$ testing, the baseline orientation selected for all $1 \mathrm{~g}$ operations is a vertical, both water and oxygen up-flow configuration. Chemical performance data indicates that sufficient gas distribution and acceptable reactor gas occlusion is achieved in this orientation in $1-\mathrm{g}(1)$.

The REGEN HX also plays an important role in maintaining VRA effectiveness and energy efficiency. The presence of gaseous oxygen and oxidation byproducts within the reactor effluent makes the analytical prediction of heat exchanger performance difficult. The accumulation of gas films or bubbles on heat transfer surfaces within the VRA heat exchangers could potentially inhibit heat transfer to the point that process 
temperatures cannot be maintained. This could affect the VRA reactor chemical performance, power consumption, and/or the VRA effluent water temperature to exceeds the temperature-tolerance of the downstream components.

A schematic of the VRAFE is shown in Figure 2. The objective of the VRAFE was to evaluate the micro gravity performance of these two critical components within the VRA. The experiment was designed as a self-contained closed loop water system that utilizes a single diluted contaminant, ethanol, for the performance evaluation. The ethanol was injected into the system to maintain an 80-150 $\mathrm{mg} / \mathrm{l}$ concentration. In addition to the ethanol, gaseous oxygen was injected into the system to provide an oxidant for the reactor operation. The experiment was designed so that it could be tested both on the ground and in a micro gravity environment. The results from both areas of testing would then be compared to determine any possible performance effects as a result of micro gravity.

Figure 2. VRAFE Simplified Functional Schematic

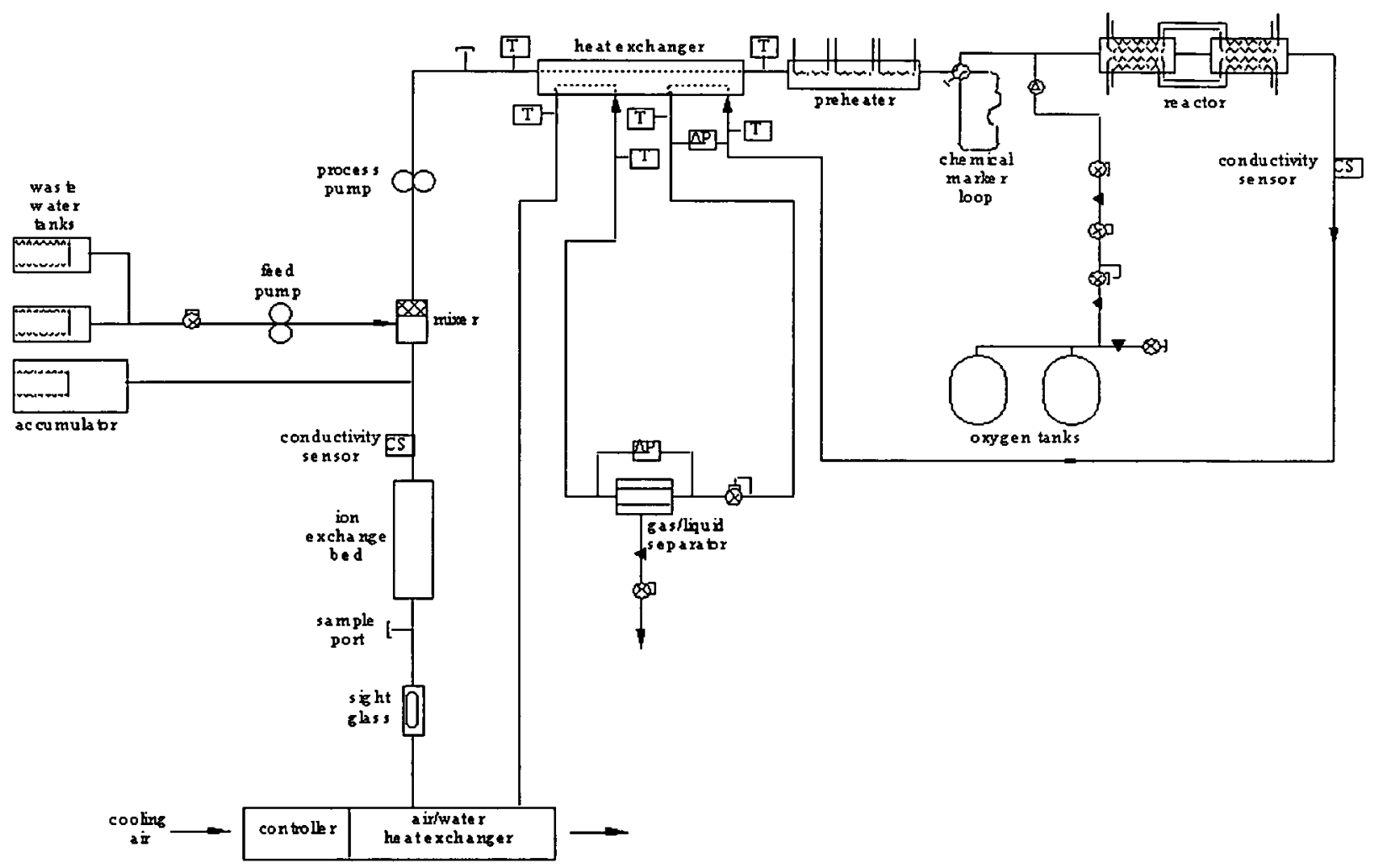

The VRAFE had two methods of evaluating the micro gravity affect on the catalytic reactor. First, water samples pulled during ground and micro gravity operation would show any affect that micro gravity had on chemical performance. Also, a residence time check was performed during micro gravity and ground operations to determine if gas occlusion within the reactor was affected by micro gravity. This residence time check was done by injecting a chemical marker upstream of the reactor and measuring the conductivity response downstream of the reactor.
VRAFE also provided comparison data for the REGEN $\mathrm{HX}$ performance. Temperature sensors were located at the inlets and outlets of the REGEN HX so that efficiency of the REGEN HX in micro gravity could be compared to ground operation.

Flow visualization ground testing of the catalytic reactor in its baseline orientation (vertical, up-flow) indicated that in $1-\mathrm{g}$, the reactor operated at a $25-35 \%$ gas occlusion level. Gas bubbles moved through the packing with in a "chugging flow", where the bubble size was controlled by 
buoyancy and capillary forces within the packing. These forces allowed bubbles from 4-6 inches long. Though this is not ideal gas distribution, chemical data showed it was sufficient for the reactor to meet it oxidation requirements by over a 3 to 1 margin.

In addition to the VRA FE test data, flow visualization testing of catalytic reactors on the $\mathrm{KC}-135$ was performed to observe the gas distribution and measure gas occlusion levels. This measurements and observation could then be compared to the 1-g test data to assess micro gravity affects on the reactor performance.

The KC-135 testing used small-scaled beds so that steady state could be reached during the short micro gravity periods aboard the KC-135 $(<20 \mathrm{sec})$. Glass beads were used as packing in several beds to help in visualizing the gas distribution and catalyst substrate was used in others to represent the expected channel sizes and surface tensions within the VRA flight reactor so that accurate occlusion measurements could be taken. Beds were run in the horizontal and vertical positions and were started at various, known gas liquid occlusion levels. Two-phase flow was only passed through the beds during micro gravity periods. During the gravity periods, the beds were isolated. The bed was determined to be at steady state occlusion based on a qualitative assessment of steady gas/liquid flow observed at the bed outlet. The beds were then isolated and taken off line. When the $\mathrm{KC}-135$ was back on the ground, the final occlusion levels were measured in each bed.

\section{CATALYTIC REACTOR RESULTS}

VRAFE RESULTS - The graph in Figure 3 shows micro gravity and ground reactor outlet conductivity after the chemical marker was injected upstream of the reactor. As can be seen from the graph, not only was the time to breakthrough of the reactor the same in micro gravity as in $1-\mathrm{g}$, but the curves have an identical shape. The magnitude difference is accounted for by slight differences in marker concentrations between the tests. These curves would indicate that the residence time, and therefore gas occlusion level, was not affected by micro gravity. Another method was also used to estimate gas occlusion levels during micro gravity testing. It was discovered that when gas bubbles move across the reactor outlet conductivity sensor, signal dropout occurs. Using this signal dropout as an indication of when gas exits the reactor and knowing the inlet gas flowrate, a "time-to-gas-breakthrough" can be calculated for when gas is initially flowed into the reactor. Since this initial breakthrough occurs before ethanol is fed to the system (no $\mathrm{O} 2$ is consumed), if it is assumed that the reactor has no gas occlusion initially, a rough estimate can be made of the of how much of the reactor is occluded with gas. Using this method, the gas occlusion for the reactor in micro gravity was estimated at $25 \%$.

Figure 3. Comparison of Ground and Flight Chemical Marker Tests

$$
\begin{array}{|l|}
\hline 0-\operatorname{cs} 7535 \text { (STS-96) } \\
\cdots \cdots \operatorname{cs} 7535 \text { (post-night ground test) }
\end{array}
$$

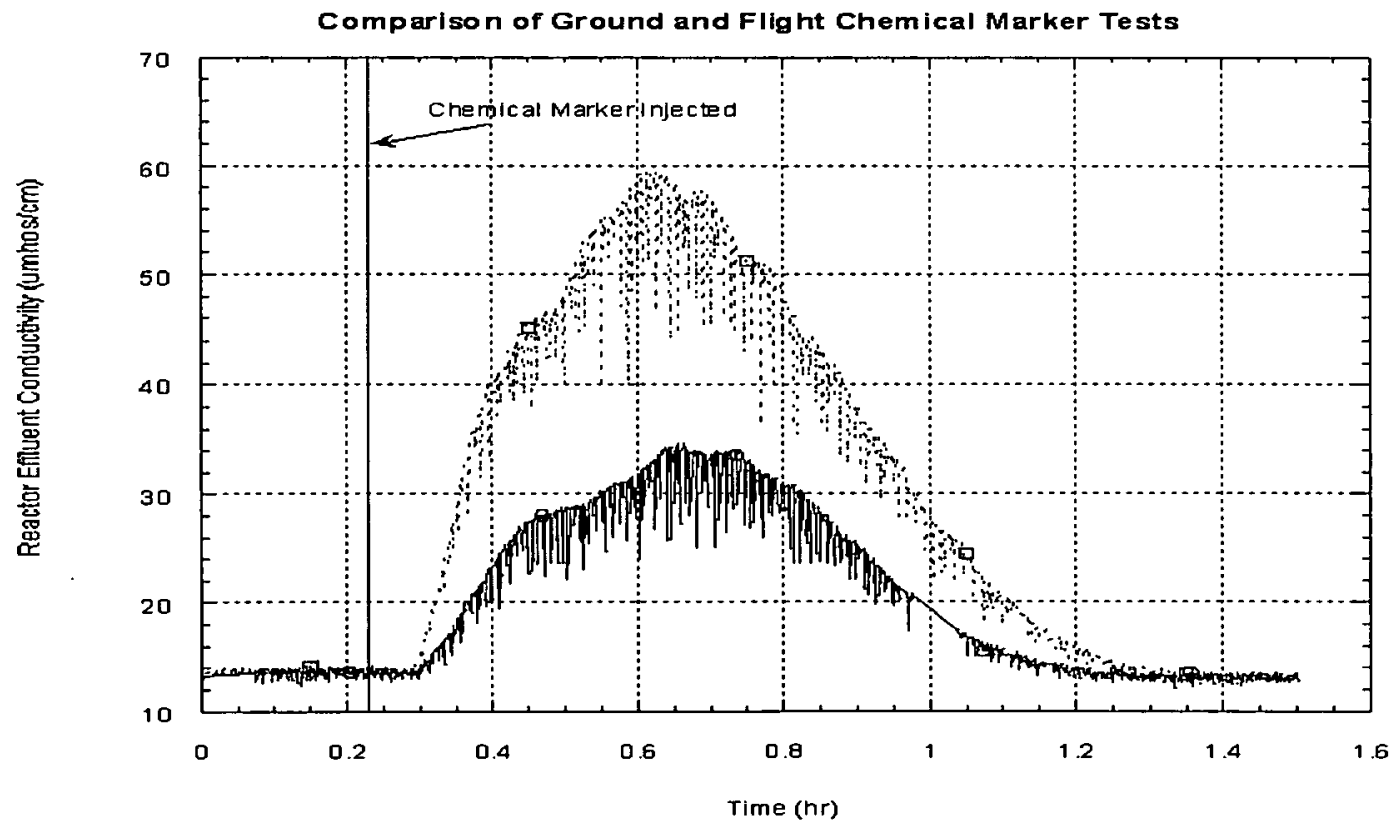


Chemical performance data of the reactor during micro gravity and $1-\mathrm{g}$ operation is summarized in Table 1 . Because of problems with the ethanol injection during flight, only 2 of the 6 samples pulled during flight provided useful results. Based on the reactor inlet and outlet concentration of ethanol and acetic acid and the known flowrate of oxygen, the percent oxygen utilization can be calculated. The test data indicates that in micro gravity, $72 \%$ of the available oxygen was utilized as compared to identical conditions in $1-\mathrm{g}$ testing where 87 $\%$ of the available oxygen was utilized (a $17 \%$ reduction in oxygen utilization due to micro gravity).

Table 1. VRAFE Reactor Chemical Performance Summary

\begin{tabular}{|l|c|c|}
\hline Parameter & $\begin{array}{c}\text { STS-96 Flight } \\
\text { Test }\end{array}$ & $\begin{array}{c}\text { Post-Flight } \\
\text { Ground Test }\end{array}$ \\
\hline & & \\
\hline Reactor Influent & & \\
\hline Ethanol (mg/l) & 145 & 144 \\
\hline Acetic Acid(mg/) & 0 & 0 \\
\hline & & \\
\hline Reactor Effluent & & 0 \\
\hline Ethanol (mg/l) & 0 & 91 \\
\hline Acetic Acid(mg/l) & 131.8 & \\
\hline & & 0.0045 \\
\hline $\begin{array}{l}\text { O2 flowrate } \\
\text { (lb/hr) }\end{array}$ & 0.0045 & \\
\hline & & $86.70 \%$ \\
\hline$\%$ O2 utilized & $72 \%$ & \\
\hline
\end{tabular}

To put this reduction in oxygen utilization into perspective, the required percent oxygen utilization to completely oxidize the expected ISS nominal organic load to the VRA is $35 \%$. Therefore, even when this $17 \%$ reduction is applied to the present baseline VRA catalyst for the ISS (a much more reactive catalyst than the one used in the VRAFE), the reactor should be able to utilize $80 \%$ of the available oxygen, allowing a significant design margin for micro gravity operation.

KC-135 RESULTS - Table 2 summarizes all the test conditions for the glass bead and catalyst substrate packed beds that were run in the horizontal position. Vertical bed testing on the KC-135 was discarded because the 1-g to 2-g gravity forces between the micro gravity periods allowed gas that was in the packed bed to rise to the top plenum of the beds (out of the packing). This affect allowed gas to collect in the top plenum, skewing gas occlusion measurement. In the horizontal position, gas rose to the top of the packing during the 1$\mathrm{g}$ to 2-g gravity periods, but was quickly redistributed throughout the packing at the beginning of the next micro gravity period by the wicking of water up through the packing channels. Therefore, the horizontal orientation was determined to be the closest representation to continuous micro gravity flow.
Table 2. KC-135 Gas Occlusion Results

\begin{tabular}{|c|c|c|}
\hline Packing & $\begin{array}{c}\text { Pre-Flight Gas } \\
\text { Occlusion (\%) }\end{array}$ & $\begin{array}{c}\text { Post-flight Gas } \\
\text { Occlusion (\%) }\end{array}$ \\
\hline $\begin{array}{c}\text { Catalyst } \\
\text { Substrate }\end{array}$ & $1 \%$ & $25 \%$ \\
\hline $\begin{array}{c}\text { Catalyst } \\
\text { Substrate }\end{array}$ & $1 \%$ & $27 \%$ \\
\hline Glass beads & $1 \%$ & $28 \%$ \\
\hline $\begin{array}{c}\text { Catalyst } \\
\text { Substrate }\end{array}$ & $30 \%$ & $34 \%$ \\
\hline Glass Beads & $30 \%$ & $31 \%$ \\
\hline $\begin{array}{c}\text { Catalyst } \\
\text { Substrate }\end{array}$ & $70 \%$ & $22 \%$ \\
\hline $\begin{array}{c}\text { Catalyst } \\
\text { Substrate }\end{array}$ & $99 \%$ & $27 \%$ \\
\hline $\begin{array}{c}\text { Catalyst } \\
\text { Substrate }\end{array}$ & $99 \%$ & \\
\hline
\end{tabular}

As can be seen in the table, gas occlusion measurements taken after the packed beds reached steady state were between $25-34 \%$. The final gas occlusion measurements were independent of the initial occlusion levels (1-99\% occluded with gas). These levels were comparable to $1 \mathrm{~g} g$ test results indicating that gas occlusion was not significantly affected by micro gravity.

The flow visualization portion of the testing was not as successful. Though water and gas could be seen moving through the packing, the short duration of flow did not allow assessment of gas bubble size or how the gas was distributed throughout the bed.

\section{REGEN HX RESULTS}

The REGEN HX inlet and outlet temperatures for VRAFE were stable and identical from ground to micro gravity testing. Therefore, micro gravity had no measurable affect on heat transfer.

\section{GLS LESSONS LEARNED}

An unexpected finding was discovered when the VRAFE GLS failed to separate gas from the reactor outlet stream during the micro gravity test. The VRAFE GLS was a two-membrane (flat sheet hydrophobic and 
hydrophilic membrane) gas trap. Flight data as well as the post-flight failure investigation determined that the GLS hydrophobic membrane failed as a result of very fine hydrophilic catalyst particles from the VRAFE reactor that had contaminated the surface of the hydrophobic membrane. These particles allowed a water layer to wick across the surface of the hydrophobic membrane and effectively block the pores from passing gas. Micro gravity magnified the failure affect since there was no gravity to free drain the water layer from the hydrophobic membrane.

Though this GLS design is not baselined for the flight VRA, a hydrophobic membrane separator is used in the VRA and other membrane separators are used throughout the ISS life support systems. As a result of the GLS failure, an assessment of all the membrane separators in the water recycling and oxygen generation systems is underway to determine if membrane separation is acceptable in each application.

\section{CONCLUSIONS}

The results from the series of tests conducted on the VRA reactor and REGEN HX on the ground, on board the $\mathrm{KC} 135$ airplane and in micro gravity environment have lead to the following conclusions:

1. Gas inclusion within the packed bed reactor is approximately $30 \%$ and is not significantly affected by micro gravity.

2. The residence time in the reactor is not affected by micro gravity.
3. The slight loss in chemical performance in the reactor is well within the design margin of the reactor.

4. The performance of the REGEN HX was unaffected by a two-phase flow in micro gravity.

The efforts identified in this paper have demonstrated that the risk mitigation activities conducted for the VRA reactor and REGEN HX have significantly reduced the operational risk to the ISS WPA. In addition, the loss of performance seen in the VRA FE GLS has result in further development and re-design of the ISS Oxygen Generation Assembly, WPA and Urine Processor Assembly phase separators.

\section{ACKNOWLEDGMENTS}

The authors would like to thank the VRAFE team at Hamilton Sundstrand Space Systems International Inc. for their long hours and hard work in designing and manufacturing the VRAFE, the VRAFE team at NASA MSFC, for their tireless efforts testing and troubleshooting the VRAFE and the team at SPACEHAB/Boeing for their integration support and expertise. The authors would specifically like to thank Layne Carter and Doug Snowdon in supporting the VRAFE and KC-135 testing and data analysis.

\section{REFERENCES}

1. Bagdigian, R.M., et. al., "Space Station Regenerative Life Support Risk Mitigation Through Microgravity Flight Experiment Demonstrations", SAE \# 961513, Presented at the $26^{\text {th }}$ International Conference on Environmental Systems, Monterey, CA, July, 1996 